\title{
Estrategias de inserción laboral en población con discapacidad mental: una revisión
}

\author{
Franco Mascayano Tapia, ${ }^{1}$ Walter Lips Castro, ' José Miguel Moreno Aguilera'
}

Actualización por temas

\section{SUMMARY}

The employability and access to the productive market are considered key elements for the full integration of the patient with mental disability. The aim of this review was to describe and analyze the scientific literature for the main employability strategies: traditional vocational rehabilitation (sheltered work and social firms) and supported employment (particularly in its mode of individualized supported employment). The results of the review suggest that individualized supported employment is the most effective approach in obtaining employment for people with mental disabilities.

Key words: Mental disability, employability, sheltered work, social firms, supported employment.

\section{INTRODUCCIÓN}

La inserción laboral y el acceso al mercado productivo son considerados elementos claves para la plena integración de la persona con discapacidad mental. ${ }^{1}$ En el contexto de la investigación de los efectos de la inserción laboral, está ampliamente difundido el reconocimiento del valor positivo del empleo como herramienta de mejoría tanto de la situación clínica como psicosocial de los afectados por una alteración mental discapacitante. La actividad laboral en los usuarios no sólo mejora su situación financiera sino que también provee una estructuración de sus actividades cotidianas, con rutinas definidas y significativas. Los usuarios con empleo poseen un sentido personal de bienestar, un buen nivel de autoeficacia e identidad social. ${ }^{2}$ Se observa, además, una mejora en la autoestima y en la autoimagen, una reducción de la sintomatología, una promoción del contacto social y un aumento de la calidad de vida de quienes trabajan. ${ }^{3,4}$ Por otra parte, también se ha demostrado que el empleo estable es un gran soporte que permite la inserción social de la persona con discapacidad mental, garantizando el acceso al ejercicio de sus derechos de ciudadano. ${ }^{5}$ Algu-

\section{RESUMEN}

La inserción laboral y el acceso al mercado productivo son considerados elementos claves para la plena integración del paciente con discapacidad mental. El objetivo de la presente revisión fue describir y analizar la literatura científica correspondiente a las principales estrategias de inserción laboral: rehabilitación vocacional tradicional (empleo protegido y empresas sociales) y empleo con apoyo (particularmente en su modalidad de empleo con apoyo individualizado). Los resultados de la revisión indican que el empleo con apoyo individualizado es el abordaje más efectivo en la obtención de empleo en personas con discapacidad mental.

Palabras clave: Discapacidad mental, inserción laboral, empleo protegido, empresas sociales, empleo con apoyo. nos autores afirman ${ }^{6}$ que una integración social efectiva del enfermo mental con discapacidad exige su incorporación al mundo productivo, su capacitación laboral y el acceso al mercado de trabajo.

A pesar de lo ya mencionado, en la actualidad las personas con discapacidad mental tienen menos posibilidades para encontrar empleo en comparación con otras condiciones discapacitantes no mentales. Al respecto, el nivel de fuerza de trabajo de dichos individuos alcanza sólo el $29 \%$, un porcentaje significativamente menor si es comparado con las personas con discapacidad física (49\%) y con la comunidad en general (74\%). ${ }^{7}$ La situación es aún peor para personas con trastorno psicótico, entre quienes sólo dos de cada 10 encuentran alguna forma de empleo. ${ }^{7}$ Algunos de los factores que explican dicha situación provienen tanto de variables individuales como socioculturales. En la dimensión personal, muchos de los usuarios con trastorno mental presentan bajos niveles de formación académica, baja productividad, reducidas habilidades sociales y laborales, síntomas deficitarios tanto afectivos como cognitivos, efectos colaterales adversos de los fármacos y escasa experiencia laboral. ${ }^{8,9}$ Adicionalmente, diversas variables ambientales

Escuela de Psicología, Facultad de Medicina, Universidad de Valparaíso, Chile

Correspondencia: Franco Mascayano. Escuela de Psicología. Universidad de Valparaíso, Av. Brasil 2140, Valparaíso, Chile. E-mail: franco.mascayano@gmail.com

Recibido: 15 de febrero de 2012. Aceptado: 13 de agosto de 2012. 
también se transforman en barreras importantes para la consecución de un empleo. Dentro de éstas se describen las dinámicas exigentes y cambiantes del mercado laboral, la falta de incentivos laborales, el disentimiento con el trabajo particular a desempeñar, el estigma público con la consiguiente discriminación tanto por parte de los empleadores como de la población en general, $y$, finalmente, la falta de programas clínicos y vocacionales que apoyen la inserción laboral. ${ }^{10-12}$

Como se mencionó anteriormente, la actividad laboral juega un rol importante en la recuperación de personas que padecen alguna alteración mental discapaciante. ${ }^{13,14}$ Sin embargo, es importante destacar que uno de los factores que predice en forma importante el éxito para lograr y mantener un trabajo es la motivación por trabajar. Al respecto, según los estudios de Perkinset et al. ${ }^{15}$ y Chuaqui, ${ }^{16}$ las personas con discapacidad mental presentan alta motivación por trabajar. Por lo tanto, es importante que toda sociedad adecuadamente organizada considere medidas que, con fundamento científico, faciliten y promuevan la empleabilidad de las personas que sufren problemas mentales con discapacidad.

Las estrategias de inserción que describiremos en el presente artículo se dividen en dos grandes categorías: 1. la rehabilitación vocacional tradicional, que se subdivide en Empleo Protegido (Sheltered work) y Empresas Sociales (Social Firms); 2. el empleo con Apoyo (Supported Employment). Dentro de esta categoría analizaremos en profundidad el modelo que en la actualidad ha mostrado mayor eficacia para la integración laboral: el Modelo de Empleo con Apoyo Individualizado (Individual placement and support).

\section{REHABILITACIÓN VOCACIONAL TRADICIONAL}

Consiste en programas diseñados para restablecer o desarrollar habilidades en aquellas personas que sufren alguna discapacidad mental. Su objetivo principal apunta tanto al logro como a la conservación de un trabajo adecuado para dichos usuarios. Se recurre a procedimientos de evaluación en etapas de pre-empleo, actividades de entrenamiento para el trabajo, consejería laboral y ayuda para la colocación laboral. Su característica principal es el paradigma "train-place", es decir, primero se prepara a la persona para un trabajo protegido o competitivo, mediante un entrenamiento vocacional, y luego se concreta la ubicación laboral de la persona rehabilitada.

1. Integración laboral mediante empleo protegido. Según el abogado Miguel Laloma García, el Empleo Protegido "es aquel que ha sido generado para personas con discapacidad en empresas ordinarias que cumplen determinadas características orientadas a facilitar la incorporación de trabajadores con discapacidad al mercado laboral" ${ }^{17} \mathrm{El}$ objetivo principal de este tipo de empleo es la realización de un trabajo productivo, la participación en operaciones de mercado y la mantención de una remuneración. ${ }^{18}$ Estos empleos han sido concebidos para personas que presentan una enfermedad mental grave, un bajo nivel de funcionamiento social y que adolezcan de una preparación suficiente como para participar en espacios laborales normalizados. Respecto a las remuneraciones, éstas sólo ocasionalmente se correlacionan con la calidad de los productos confeccionados o con el logro de las tareas realizadas y suelen ser de baja cuantía. ${ }^{19}$ En el empleo protegido se engloban abordajes de fomento laboral como Clubhouses, ${ }^{20}$ programas de colocación laboral diversificada ${ }^{21}$ y otras estrategias de fomento vocacional incluidas en los programas de rehabilitación psiquiátrica. $^{22} \mathrm{El}$ objetivo de estas estrategias es doble: por un lado, asegurar un empleo remunerado a aquellas personas con discapacidad que, independientemente de la causa, no puedan acceder a un empleo normalizado; y por otro lado, intentar preparar a estas personas para un posible acceso al mercado ordinario. Generalmente la plantilla de trabajadores está configurada mayoritariamente por personas con discapacidad, con el fin de facilitar el desarrollo personal y social en un ambiente de comprensión y pertenencia. ${ }^{23}$ Sin embargo, las estrategias de Empleo Protegido han mostrado limitaciones a la hora de dar cumplimiento al objetivo planteado anteriormente. Al respecto, frecuentemente los trabajadores no realizan un trabajo productivo, permaneciendo en su actividad laboral más bien por razones sociales que por su productividad. Además frecuentemente no se logra la promoción desde la condición protegida al empleo normalizado. ${ }^{18}$

Con relación al estudio de la efectividad de estas intervenciones se han realizado encuestas aplicadas en sectores de la población norteamericana, las cuales han demostrado que las tasas de empleo se encuentran entre el 30 y el $40 \%$ en aquellas personas que han participado de algún programa de empleo con apoyo. ${ }^{24}$ Macias et al. ${ }^{25}$ mencionan que varios clubhouses presentaron en promedio un $19.6 \%$ de miembros activos participando en empleos transitorios y un $17.5 \%$ en empleos competitivos independientes. Por otra parte, en un estudio reciente en el que se comparó el trabajo de un Clubhouse con una intervención de empleo con apoyo llamado "Programa de Tratamiento Asertivo Comunitario (ACT, en ingles)", se obtuvo resultados semejantes respecto de la cantidad de horas semanalmente trabajadas e ingresos mensuales. ${ }^{26}$ No obstante, en la revisión realizada por McKay, Johnsen y Stein, ${ }^{27}$ se reportó que en el período 1998-2001, alrededor de 1702 miembros de 17 clubhouses diferentes consiguieron empleo mayoritariamente de tipo transicional. Además, se observó que los usuarios percibieron sueldos más bajos que los establecidos por el mercado. A pesar de que el impacto en la empleabilidad de los empleos protegidos no cuente con evidencia sólida que 
lo avale, tales intervenciones poseen beneficios de índole terapéutica que es relevante mencionar:28-31

a) Reducción de recaídas y hospitalizaciones;

b) Disminución de las problemáticas con el sistema judicial;

c) Aumento de la autonomía, la funcionalidad y la autoeficacia personal;

d) Mayor sensación de bienestar, que radicaría en la formación de redes de amistad y apoyo.

2. Integración Laboral en Empresas Sociales. La Asociación Europea de Empresas Sociales (CEFEC, por sus siglas en inglés) las define como una empresa ordinaria para emplear a personas con discapacidad u otras desventajas en el mercado del trabajo. ${ }^{32}$ Las empresas sociales se originaron en Italia en la década de los 1960, y desde entonces han proliferado en Europa, Norteamérica y Asia, principalmente. Estas empresas se caracterizan por un proceder solidario, permitiendo el acceso a un empleo para personas con discapacidad. Las plantillas laborales están conformadas por un 20 a 50\% de empleados con discapacidad y las remuneraciones se calculan sobre una tasa base de productividad. ${ }^{7}$ En estas instituciones se intenta integrar el factor económico con el factor social. En dichas empresas se evalúan las limitaciones y necesidades de cada individuo. A partir de estas evaluaciones se planifica un programa de inserción laboral personalizado, cuyo objetivo es la adquisición de habilidades suficientes para lograr el acceso al mundo laboral. Para lograr esto último se ha comprobado que la estrategia más eficaz es la formación y el aprendizaje en un entorno laboral común. Por el contrario, se ha demostrado que la mera formación teórica no resulta útil para aprender un oficio, lo que podría generar frustración en el individuo. ${ }^{33}$

Según nos indica Peter Stadler, ${ }^{34}$ miembro de CEFEC, hay actualmente en Alemania más de 600 empresas sociales, cada una con una plantilla de trabajadores en las que entre el 25 a 50\% de ellos tiene alguna discapacidad. Según el estudio de Smit, van Genabeek y Mike Klerkx, ${ }^{35}$ en el que se recogieron datos de cinco países europeos (Alemania, Italia, Béliga, Reino Unido y Suecia), se observó que la mayoría de las empresas sociales ofrecen salarios de mercado y puestos laborales permanentes para sus usuarios. Además, gran parte de estas empresas sociales contratan trabajadores con algún grado de discapacidad mental. Según dichos autores, la actividad laboral conjunta entre personas con discapacidad y sin discapacidad mental estimula la integración social de aquellos que presentan discapacidad, potenciando a su vez su desempeño laboral. Similares conclusiones se han observado en otras investigaciones realizadas en países europeos como España, ${ }^{36}$ Finlandia, ${ }^{37}$ Portugal $^{38}$ e Irlanda. ${ }^{39}$ Lo mismo se ha descrito en empresas sociales constituidas en los Estados Unidos, Australia, Nueva Zelanda, Japón y Korea. ${ }^{40}$ Sin embargo, dado que los estudios antedichos no son experimentales ni cuasiexperimentales, sus resultados deben observarse con cautela debido a la falta de contrastación con otros modelos de inserción laboral.

Por último, al igual que en el empleo protegido, algunos autores señalan que el involucramiento en empresas sociales genera beneficios terapéuticos, incluyendo la disminución de la demanda de los servicios de salud mental, la reducción del aislamiento social y el incremento tanto de la autoconfianza como de la motivación por parte del usuario. ${ }^{41,42}$ Lo anterior redunda en una mejor calidad de vida de los usuarios. Al respecto, en un estudio cualitativo realizado por Svanberg, Gumley y Wilson, ${ }^{43}$ se identificaron factores vivenciales positivos que repercutieron favorablemente en el bienestar de los trabajadores. Entre dichos factores se hace mención a: la estructura flexible del trabajo en las empresas sociales, el significado y la diversidad de las actividades laborales, el sentido de integración a un grupo social y, finalmente, la generación de liderazgos.

Empleo con apoyo. Se basa en el paradigma "place-train", es decir, se promueve con prontitud la colocación laboral competitiva de personas que presenten alguna discapacidad. Por lo tanto se les ofrecen procesos de entrenamiento y apoyo de acuerdo a las necesidades que surjan durante sus actividades laborales. El Modelo de Empleo con Apoyo es creado en los Estados Unidos en la década de 1990, gracias al trabajo de Robert Drake y Deborah Becker, ${ }^{44,45}$ a partir de los conceptos de integración y normalización sociales. Fue diseñado para favorecer la integración laboral de aquellas personas con discapacidad que estén motivadas para trabajar.

Según el psicólogo estadounidense Gary Bond, basándose en el Rehabilitation Act Amendments, el empleo con apoyo se caracteriza por lo siguiente: ${ }^{46}$

- La presencia de la figura del Preparador Laboral. Se trata de un profesional que acompaña a la persona con discapacidad en su lugar de trabajo, ayudándole en el proceso de entrenamiento laboral. Esta figura es fundamental ya que proporciona el apoyo que facilitará la integración. Además debe fomentar que la persona se implique en su entrenamiento, en resolver los problemas que puedan surgir y en conseguir que los agentes relacionados con la persona (familia, amigos $u$ otros) participen también de este proceso.

- Apoyo en el puesto de trabajo. Esta es una característica clave del modelo ya que permitirá que la persona pueda acceder y mantenerse en el empleo. Existen distintos niveles de apoyo que dependerán de las necesidades de la persona, de las necesidades del entorno, y que variarán en función del momento. Dentro de estos tipos de apoyo están el apoyo intermitente, el apoyo limitado, el apoyo extenso y el apoyo generalizado. Se ha observado que el colectivo de personas con algún trastorno mental crónico generalmente suele necesitar un apoyo de tipo extenso y generalizado. ${ }^{47}$ 
- Planificación centrada en la persona. Todo el proceso de empleo con apoyo está basado en los intereses, motivaciones y habilidades de las personas, haciéndolos partícipes del mismo. Por lo tanto se debe hacer una valoración individualizada, atendiendo principalmente a cómo la persona se desenvuelve en su entorno más que a las dificultades que presenta en el mismo.

- Puestos de trabajo en empresas ordinarias en condiciones de igualdad. La persona debe ser integrada en puestos de trabajo dentro de empresas competitivas en el mercado laboral en las mismas condiciones que el resto de sus compañeros.

Respecto de las formas de implementación del trabajo con apoyo, la más estudiada y estandarizada para personas que sufren discapacidad mental es la colocación laboral individualizada con apoyo (IPS: Individualized Placement and Support). Las características principales del IPS son las siguientes: a) la presencia de agencias proveedoras de servicios para la consecución de un empleo competitivo, b) la búsqueda rápida de empleo que permita ayudar a los clientes a obtener puestos laborales con relativa prontitud, c) una interacción entre la agencia y los clientes para la ubicación de un trabajo concordante con las preferencias, fortalezas y experiencia laboral previa del cliente, d) un constante apoyo y seguimiento por parte de la agencia respecto del desempeño laboral del cliente, y, finalmente, e) una estrecha vinculación entre la agencia y el tratamiento psiquiátrico de cada cliente. Bond et al. ${ }^{48}$ determinaron que dichas características son predictores positivos para la inserción laboral en personas con discapacidad mental.

Según diversos estudios de tipo clínico aleatorio y controlado (ECA) el IPS generaría los mejores resultados en materia de empleo en personas con discapacidad mental. Respecto del costo de su implementación, no sólo es relativamente bajo, además es de sencilla aplicación. ${ }^{49}$ En un estudio realizado por Drake et al..$^{50}$ se contrastó el IPS con dos programas de inserción laboral tradicional. Los resultados mostraron que los clientes del programa IPS obtuvieron más empleos competitivos durante un seguimiento de 18 meses. En resumen, de este estudio se concluye que el IPS es un sistema de apoyo más efectivo que los otros programas tradicionales. En otro estudio de Drake et al., ${ }^{51}$ nuevamente se observan resultados que indican una mayor efectividad del IPS en contraste con los sistemas de inserción tradicionales, tanto en la obtención de empleos competitivos como en el número de horas semanales trabajadas.

En otra investigación realizada por Lehman et al., ${ }^{52}$ se evaluó la eficacia del modelo IPS en relación a programas de rehabilitación psicosocial habitual para inserción laboral en personas con enfermedades mentales graves. Los individuos en el programa IPS mostraron mejores indicadores de empleabilidad que el grupo de comparación (42\% vs. 11\%, respectivamente). Respecto de datos específicos relativos a la obtención de empleo competitivo se observó una dife- rencia notoria entre el IPS y los otros programas (27\% vs. $7 \%)$. Por su parte, el equipo de Mueser et al. ${ }^{53}$ comparó tres métodos de inserción profesional en población psiquiátrica, entre ellos el modelo IPS. Los usuarios de IPS tuvieron una mayor empleabilidad competitiva (73.9\%) que los usuarios de los otros dos métodos (18.2\% y $27.5 \%$ ). Con respecto a las remuneraciones obtenidas, el $73.9 \%$ de los usuarios del programa IPS lograron mejores sueldos que los clientes de las otras dos estrategias.

En el año 2006, Latimer et al., ${ }^{54}$ en un ECA, buscaron determinar la eficacia del modelo IPS en población canadiense. Durante los 12 meses de seguimiento, el 47\% de los clientes en el grupo IPS obtuvo al menos un empleo competitivo frente al 18\% del grupo control. Cabe indicar que el modelo IPS ha sido extensamente difundido en los servicios de salud mental de Canadá. ${ }^{55,56}$

En otro ECA, Burns et al..$^{57}$ realizaron un estudio multicéntrico en seis países europeos (Reino Unido, Alemania, Italia, Bulgaria, Suiza y Holanda). Dichos autores demostraron la eficacia del IPS frente a otros servicios modelos, respecto a la capacidad para conseguir empleo. En un análisis posterior publicado por los mismos autores se concluyó que las personas que consiguieron empleo presentaron un mejor funcionamiento global, menor cantidad y severidad de síntomas y menor nivel de discapacidad. ${ }^{58}$ Por último, Bond et al., ${ }^{59}$ también en un ECA, compararon el IPS y el enfoque de colocación diversificada (ECD). Se encontró que el modelo IPS es superior al ECD en el logro de empleabilidad (75\% vs. 33\%).

En un ECA realizado por Wong et al., ${ }^{60}$ se aplicó el IPS en usuarios de un hospital de Hong Kong. En comparación con los participantes en programas de rehabilitación convencionales, los usuarios del IPS presentaron mayores posibilidades para conseguir trabajos competitivos, remuneraciones más altas, mayor número de días trabajados y una mayor capacidad para mantener su empleo. Por otra parte, Killickey et al. ${ }^{61}$ evaluaron la utilidad del IPS aplicado en personas con primer episodio de psicosis. Se encontró que el grupo IPS obtuvo mejores resultados en la adquisición de empleo, en horas trabajadas por semana y en tiempo de permanencia en el trabajo.

En otro ECA, Twamley et al. ${ }^{62}$ evaluaron el nivel de empleabilidad en personas de 45 o más años de edad, diagnosticadas con esquizofrenia o trastorno esquizoafectivo. Se comparó el IPS con un programa de rehabilitación vocacional convencional. El programa IPS impactó positiva y significativamente en el logro de un empleo competitivo, el número de semanas de trabajo y la obtención de mejores salarios y una mejor calidad de vida. En otro estudio semejante realizado posteriormente por el mismo equipo se observaron resultados similares. ${ }^{63}$

Por último, en el año 2010 se públicaron los resultados del ECA de Howard et al., ${ }^{64}$ en donde se comparó el modelo IPS con los servicios vocacionales tradicionales, en Londres. En este estudio no hubo diferencias significativas entre gru- 
Estrategias de inserción laboral en población con discapacidad mental

pos comparados respecto de la adquisición de empleo competitivo. Resulta importante resaltar que ésta es la primera investigación que refuta la efectividad del modelo IPS.

\section{DISCUSIÓN}

En la realización de este artículo hemos revisado diversas estrategias dirigidas para la inserción laboral de personas con alteraciones psiquiátricas discapacitantes.

En relación a los enfoques más tradicionales de inserción laboral, los estudios revisados señalan un impacto medianamente positivo de tales intervenciones, con mejores resultados en los indicadores clínicos (remisión de síntomas, bienestar subjetivo, sentido de pertenencia social, reducción de hospitalizaciones, etc.), en comparación a las dimensiones laborales propiamente tales (empleo competitivo, sueldos de mercado, horas semanales trabajadas, etc.).

En el caso de los empleos protegidos, no existe evidencia sólida que avale a este tipo de estrategia en materia de empleabilidad, menos aún de tipo competitivo. Además, generalmente los trabajos adquiridos son transitorios ${ }^{65}$ y los sueldos se sitúan frecuentemente por debajo de la media definida por el mercado laboral. ${ }^{19}$ Siguiendo el planteamiento del sociólogo Jorge Chuaqui coincidimos en que el empleo protegido no resuelve satisfactoriamente el problema de la inserción laboral en personas con discapacidad mental. Al respecto cabe destacar que debido a que los usuarios se desenvuelven en un ambiente "artificial" quedan privados de la interacción social propia de un contexto laboral ordinario. Sumado a lo anterior, las bajas remuneraciones y la poca exigencia laboral por metas hacen del empleo protegido una intervención insuficiente para la integración social completa del individuo. ${ }^{16}$ Sin embargo, en cuanto a los beneficios de tipo terapéutico que redundan en una mejor calidad de vida, los empleos protegidos tienen un rol fundamental. Nos parece importante destacar que dicha estrategia debería considerar planificarse en un tiempo acotado y con un objetivo específico que permita al usuario enfrentarse adecuadamente a las condiciones reales de superviviencia económica.

Respecto de las empresas sociales, se han observado resultados positivos en lo concerniente a la obtención de empleos permanentes, con sueldos de mercado y en un ambiente laboral que permite una mejor integración entre los usuarios y los demás trabajadores. Las experiencias reportadas por diferentes países mediante entrevistas y encuestas demuestran la utilidad de este enfoque para facilitar la inserción laboral en personas con discapacidad mental. Sin embargo, la sistematización de indicadores laborales a través de estudios experimentales que permitan comparar este abordaje con otras intervenciones continúa siendo una tarea pendiente. ${ }^{66}$ Además de los beneficios estrictamente laborales, es destacable que las empresas sociales fomentan la mejoría en una serie de aspectos clínico-terapéuticos de los usuarios que trabajan en ellas. Se ha observado una mayor autonomía, funcionalidad, autoeficacia y empoderamiento en los usuarios. Dado que las empresas sociales proporcionan un entorno integrado con la comunidad, permitiendo la interacción de los usuarios con personas sin discapacidades, facilitan el derribamiento de prejuicios, la reducción de los procesos de estigmatización y autoestigmatización y, por ende, aumentan la comprensión de la enfermedad mental en la comunidad. ${ }^{67}$

Finalmente, los resultados de todos los estudios revisados para el presente artículo nos permiten concluir que el enfoque de inserción laboral que ha mostrado mayor eficacia en la obtención de empleo competitivo para personas con discapacidad mental es el empleo con apoyo, específicamente el IPS. En comparación con los demás abordajes vocacionales tradicionales (empleos protegidos y empresas sociales) el IPS permite mayores tasas de obtención de empleos, mejores remuneraciones, más horas semanales trabajadas y mayor tiempo de permanencia en el trabajo conseguido. ${ }^{68}$ Además, existe evidencia que demuestra los efectos positivos que la intervención IPS produce en algunos indicadores clínicos como el nivel de funcionalidad global social, la presencia de recaídas, la calidad de vida y la autoestima. ${ }^{21}$ Tales hallazgos resultan particularmente significativos por dos razones: 1. por la existencia de evidencia clínica de primer nivel que apoya la efectividad del enfoque IPS y 2. su aplicación en diferentes contextos socioculturales (EU, Europa y China), con resultados semejantes.

A pesar de la evidencia reportada respecto al empleo con apoyo, particularmente el IPS, observamos que tanto su implementación como la investigación de su efectividad en Latinoamérica es prácticamente nula. Esto es válido incluso para países como la Argentina y el Brasil, que se rigen oficialmente por modelos de psiquiatría comunitaria. ${ }^{69}$ Dentro de los escasos estudios publicados, destacamos el realizado por el equipo uruguayo de Pardo et al., quienes implementaron un programa de empleo con apoyo en una cooperativa de trabajo. En este caso el 57\% de los usuarios pudo realizar con continuidad el programa de aprendizaje y desarrollo. ${ }^{70}$ Otro estudio publicado en población latinoamericana es el de Hernández et al., ${ }^{71}$ quienes aplicaron un programa de empleo con apoyo en usuarios mexicanos. Los autores constataron que 11 de los 24 participantes consiguieron un trabajo regular. Es importante señalar que ambos estudios citados son de tipo no experimental, por lo que los resultados no son de primer nivel de evidencia clínica según los cánones de la medicina basada en evidencia. ${ }^{72}$ Por lo tanto, consideramos importante la realización de ECAs relativos a programas de inserción laboral en personas con discapacidad mental dentro de Latinoamérica para fundamentar la gestión sanitaria correspondiente.

\section{REFERENCIAS}

1. Verdugo MA, De Urríes FB. Hacia la integración plena mediante el empleo. Actas del VI Simposio Internacional de Empleo con Apoyo. Salamanca: Instituto Universitario de Integración en la Comunidad; 2002. 
2. Tsang HW, Fong MW, Fung MT, Corrigan PW. Reducing employers' stigma by supported employment. En: Lloyd C (ed.). Vocational rehabilitation and mental health. Oxford: John Wiley \& Sons; 2010.

3. Ackerman GW, McReynolds CJ. Strategies to promote successful employment of people with psychiatric disabilities. J Appl Rehabil Couns 2005;36:35-40.

4. Becker DR, Drake RE, Naughton WJ. Supported employment for people with co-occurring disorders. Psychiatr Rehabil J 2005;28:332-338.

5. López M, Laviana M, Álvarez F, González S et al. Actividad productiva y empleo de personas con trastorno mental severo. Algunas propuestas de actuación basadas en la información disponible. Rev Asoc Esp Neuropsiq 2004;89:31-65.

6. Boardman J, Grove B, Perkins R, Shepherd G. Work and employment for people with psychiatric disabilities. Br J Psychiatry 2003;182:467-468.

7. Mental Health Council of Australia. Let's get to work. A national mental health employment strategy for Australia. Melbourne: 2007.

8. Cook JA. Employment barriers for persons with psychiatric disabilities: update of a report for the president's commission. Psychiatr Serv 2006;57:1391-1405.

9. Ferdinandi A, Yootanasumpun V, Pollack S, Bermanzohn P. Rehab rounds: predicting rehabilitation outcome among patients with schizophrenia. Psychiatr Serv 1998;49:907-909.

10. Henry AD, Lucca AM. Facilitators and barriers to employment: the perspectives of people with psychiatric disabilities and employment service providers. Work 2004;22:169-182.

11. Marwaha S, Johnson S. Schizophrenia and employment. Soc Psychiatry Psychiatr Epidemio 2004;39:337-349.

12. Tsang HW, Tam $P$, Chan F, Cheung WM. Stigmatizing attitudes towards individuals with mental illness in Hong Kong: implications to their recovery. J Community Psychol 2003;31:383-396.

13. Borg M, Kristiansen K. Working on the edge: the meaning of work for people recovering from severe mental distress in Norway. Disabil Soc 2008;23(5):511-523.

14. Shepherd G, Boardman J, Slade M. Making recovery a reality. Londres: Sainsbury Centre for Mental Health; 2008.

15. Perkins R, Farmer P, Litchfield P. Realizing ambitions: better employment support for people with a mental health condition. Londres: Department for Work and Pensions; 2009.

16. Chuaqui J. Reintegración laboral de personas con esquizofrenia: tarea incumplida. Revista de ciencias sociales 2008;53:249-264.

17. Laloma M. Empleo protegido en España. Análisis de la normativa legal y logros alcanzados. Madrid: Cinca; 2007.

18. Rodríguez F, García MC, Rodríguez MN. La integración laboral de las personas con trastorno mental grave. Una cuestión pendiente. Psiquis 2004;25(6):26-43.

19. Chuaqui J. El estigma de la esquizofrenia. Ciencias Sociales 2005;2(1):45-66.

20. Dincin J. Psychiatric rehabilitation. Schizophrenia Bull 1975;1:131-147.

21. Bond GR, Drake RE, Becker DR, Mueser KT. Effectiveness of psychiatric rehabilitation approaches for employment of people with severe mental illness. J Disabil Policy Stud 1999;10:18-52.

22. Waghorn G, Lloyd C. The employment of people with mental illness. AeJAMH 2005;4:1-43.

23. Díaz D, Chacón S. Proceso de inserción laboral de personas con esquizofrenia. Análisis desde una institución de apoyo. Revista Psicología 2006;1:27-55.

24. Rutman ID, Armstrong K. A comprehensive, national evaluation of transitional employment programs for the psychiatrically disabled. Philadelphia: Matrix Research Institute; 1985.

25. Macias C, Jackson R, Schroeder C, Wang Q. What is a clubhouse? Report on the ICCD 1996 survey of USA clubhouses. Community Ment Health J 1999;35:181-190.

26. Schonebaum AD, Boyd JK, Dudek KJ. A comparison of competitive employment outcomes for the clubhouse and PACT models. Psychiatr Serv 2006;57(10):1416-1420.
27. McKay CE, Johnsen M, Banks S, Stein R. Employment transitions for clubhouse members. Work 2006;26:67-74.

28. Di Masso J, Avi-Itzhak T, Obler DR. The Clubhouse model: An outcome study on attendance, work attainment and status, and hospitalization recidivism. Work 2001;17:23-30.

29. Johnson J, Hickey S. Arrests and incarcerations after psychosocial program involvement: Clubhouse vs. Jailhouse. Psychiatr Rehabil J 1999;23:66-70.

30. Warner R, Huxley P, Berg T. An evaluation of the impact of Clubhouse membership on quality of life and treatment utilization. Int $\mathrm{J}$ Soc Psychiatry 1999;45(4):310-320.

31. Leff HS, Wise M. Measuring service system implementation in a public mental health system through provider descriptions of employment service need and use. Psychosoc Rehabil J 1995;18(4):51-64.

32. Spear R. National profiles of work integration social enterprises: United Kingdom. Working papers series. Liège: EMES European Research Network; 2002.

33. Aiken $\mathrm{M}$. What is the role of social enterprise in finding, creating and maintaining employment for disadvantaged groups? Londres: Office of the Third Sector; 2007.

34. Stadler P. Successful structures to develop social firms in Germany -What can we learn for a development on the European level? Praga: CEFEC Conference; 2010.

35. Smit A, van Genabeek J, Klerkx M. Work integration social enterprises. European experiences with social economy. TNO: Hoofddorp; 2008.

36. Vidal I, Claver N. Work Integration social enterprises in Spain. EMES working papers series. Liège: EMES European Research Network; 2004.

37. Pättiniemi P. Work integration social enterprises in Finland. Working papers series. Liège: EMES European Research Network; 2004.

38. Perista H, Nogueira S. Work integration social enterprises in Portugal. Working papers series. Liège: EMES European Research Network; 2004.

39. $\mathrm{O}^{\prime}$ Hara $\mathrm{P}, \mathrm{O}^{\prime}$ Shaughnessy $\mathrm{M}$. Work integration social enterprises in Ireland. Working papers series. Liège: EMES European Research Network; 2004.

40. Warner R, Mandiberg J. An update on affirmative businesses or social firms for people with mental illness. Psychiatr Serv 2006;57(10):1488-1492.

41. McKeown K, O'Brien T, Fitzgerald G. Vocational rehabilitation and mental health: the European project on mental health in Ireland 19891991: Azimuth: Evaluation Report Summary 1; 1992.

42. Lanctôt $N$, Durand MJ, Corbière M. The quality of work life of people with severe mental disorders working in social enterprises: a qualitative study. Qual Life Res (en prensa).

43. Svanberg J, Gumley A, Wilson A. How do social firms contribute to recovery from mental illness? A qualitative study. Clin Psychol Psychot 2010;17(6):482-496.

44. Drake RE, Becker DR, Anthony WA. A research induction group for clients entering a mental health center research project. Hosp Community Psych 1994;45:487-489.

45. Drake RE, Becker DR, Biesanz JC, Torrey WC et al. Rehabilitative day treatment vs. supported employment: I. vocational outcomes. Community Ment Hlt J 1994;30:519-532.

46. Bond GR. Supported employment: evidence for an evidence-based practice. Psychiatr Rehabil J 2004;27:345-359.

47. Lloyd C. Evidence-based supported employment. En: Lloyd C (ed.). Vocational rehabilitation and mental health. Oxford: Wiley Blackwell; 2010.

48. Bond GR, Resnick SG, Drake RE, Xie H et al. Does competitive employment improve nonvocational outcomes for people with severe mental illness? J Consult Clin Psychol 2001;69:489-501.

49. Bond GR, Drake RE, Becker DR. An update on randomized controlled trials of evidence-based supported employment. Psychiatr Rehabil J 2008;31:280-290.

50. Drake RE, McHugo GJ, Anthony WA, Clark RE. The New Hampshire study of supported employment for people with severe mental illness. J Consult Clin Psychol 1996;64:391-399.

51. Drake RE, McHugo GJ, Bebout RR, Becker DR et al. A randomised 
clinical trial of supported employment for inner-city patients with severe mental disorders. Arch Gen Psychiat 1999;56:627-633.

52. Lehman AF, Goldberg R, Dixon LB, McNary S et al. Improving employment outcomes for persons with severe mental illnesses. Arch Gen Psychiat 2002;59:165-172.

53. Mueser KT, Clark RE, Haines M, Drake RE et al. The Hartford study of supported employment for persons with severe mental illness. J Consult Clin Psychol 2004;72:479-490.

54. Latimer E, Lecomte T, Becker D, Drake RE. Generalisablity of the individual placement and support model of supported employment: Results of a Canadian randomised controlled trial. Brit J Psychiat 2006;189:65-73.

55. Corbiere $\mathbf{M}$, Lancto $\mathbf{t} \mathbf{N}$, Lecomte $\mathrm{T}$, Latimer E. A pan-Canadian evaluation of supported employment programs dedicated to people with severe mental disorders. Community Ment Health J 2009;46:1-12.

56. Menear M, Reinharz D, Corbière $M$, Houle $N$ et al. Organizational analysis of Canadian supported employment programs for people with psychiatric disabilities. Soc Sci Med 2011;72(7):1028-1035.

57. Burns T, Catty J, Becker T, Drake RE et al. The effectiveness of supported employment for people with severe mental illness: a randomized controlled trial. Lancet 2007;370:1146-1152.

58. Burns T, Catty J, White S, Becker T et al. The impact of supported employment and working on clinical and social functioning: Results of an international Study of individual placement and support. Schizophr Bull 2009;35(5):949-958.

59. Bond GR, Salyers MP, Dincin J, Drake RE et al. A randomized controlled trial comparing two vocational models for persons with severe mental illness. J Consult Clin Psychol 2007;75:968-982.

60. Wong KK, Chiu R, Tang B, Mak D et al. A randomized controlled trial of a supported employment program for persons with long-term mental illness in Hong Kong. Psychiatr Serv 2008;59:84-90.
61. Killickey E, Jackson HJ, McGorry PD. Vocational intervention in firstepisode psychosis: individual placement and support $\mathrm{v}$. treatment as usual. Br J Psychiatry 2008;193:114-120.

62. Twamley EW, Narvaez JM, Becker DR, Bartels SJ et al. Supported employment for middle-aged and older people with schizophrenia. Am J Psychiatr Rehabil 2008;11:76-89.

63. Twamley EW, Vella L, Burton CZ, Becker DR et al. The efficacy of supported employment for middle-aged and older peoplewith schizophrenia. Schizophr Res (en prensa).

64. Howard LM, Heslin M, Leese M, McCrone P et al. Supported employment: randomised controlled trial. Br J Psychiatry;196:404-411.

65. Macias C, Kinney R, Rodica C. Transitional employment: An evaluative description of Fountain House practice. J Vocat Rehabil 1995;5:151-158.

66. López M. El empleo y la recuperación de personas con trastornos mentales graves. La experiencia de Andalucía. Norte Salud Mental 2010;36(8):11-23.

67. Secker J, Dass S, Grove B. Developing social firms in the UK: a contribution to identifying good practice. Disabil Soc 2003;18(5):659-674.

68. Campbell K, Bond GR, Drake RE. Who benefits from supported employment: a meta-analytic study. Schizophr Bull 2011;37(2):370-380.

69. Caldas de Almeida J, Cohen A (eds.). Innovative mental health programs in Latin America and the Caribbean. Washington DC: PAHO; 2008.

70. Pardo V, Del Castillo R, Blanco M, Etchart M. Descripción y evaluación de un programa de rehabilitación laboral para trastornos mentales. Rev Psiquiatr Urug 2005;69(2):111- 126.

71. Hernández J, Peralta J, Ruiz M, Angulo L et al. Rehabilitación laboral de las personas con esquizofrenia. Rev Mex Med Fis Rehab 2010;22(4):108-112.

72. Brownson RC, Gurney JG, Land GH. Evidence-based decision making in public health. J Public Health Manag Pract 1999;5:86-97. 